\title{
Single-centre evaluation and staging of rectal carcinoma on a 3-Tesla magnetic resonance imaging and correlation with histological profile
}

\author{
Jandos Amankulov ${ }^{1,2 D, E}$, Galiya Akhmetova ${ }^{1 C, D, E, F}$, Dias Toleshbaev,2B,D , Zhamilya Zholdybay ${ }^{2 A}$,

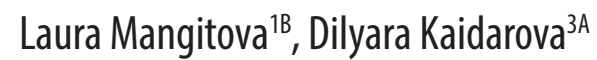 \\ 'Department of Radiology and Nuclear Medicine, Kazakh Institute of Oncology and Radiology, Kazakhstan \\ 2Department of Visual Diagnostics, Asfendiyarov Kazakh National Medical University, Kazakhstan \\ ${ }^{3}$ Department of Medical Oncology, Kazakh Institute of Oncology and Radiology, Kazakhstan
}

\section{Abstract}

Purpose: To assess magnetic resonance imaging (MRI) features of rectal carcinoma in correlation with pathology, and to evaluate the diagnostic accuracy of 3-Tesla MRI.

\begin{abstract}
Material and methods: A total of 86 patients were included. 3T MR imaging was conducted pre-operatively, with imaging data correlated to pathology results. High-resolution, 2-dimensional, T2-weighted fast-spin echo sequences in the sagittal, axial, and coronal planes used to tumour staging. Diffusion-weighted images were used to increase the accuracy of tumour evaluation.

Results: Rectal carcinoma was staged as T3 in $45.3 \%$ of all patients $(n=39)$, without involvement of the mesorectal fascia in $31.4 \%(n=27)$, and with a possible or obvious invasion in $14 \%$ of patients $(n=12)$. The diagnostic accuracy of 3T MRI was $97.6 \%$ for the T1 stage, $92.1 \%$ for T2, $89 \%$ for T3, and $90 \%$ for T4 tumours. MR-derived extramural vascular invasion (EMVI) was found in $16.2 \%(n=14)$, with an estimated diagnostic accuracy of $95 \%$. Diffusion-weighted images and apparent diffusion coefficient were estimated for the different histology types of rectal carcinoma. The average apparent diffusion coefficient for adenocarcinoma was $0.846 \pm 0.17$, for mucinous adenocarcinoma it was $1.17 \pm 0.08$, and for signet cell and squamous carcinomas it was $0.91 \pm 0.11$ and $0.796 \pm 0.21 \mathrm{~mm}^{2} / \mathrm{s}$, respectively. Conclusions: 3T MRI enables high levels of diagnostic accuracy in local rectal carcinoma staging, including assessment of mesorectal fascia infiltration and EMVI-status with high accuracy.
\end{abstract}

Key words: staging, magnetic resonance imaging, rectal carcinoma, tumour phenotype.

\section{Introduction}

Colorectal carcinoma (CRC) is the fourth most frequently diagnosed malignancy, and the second leading cause of cancer-related deaths in developed countries. Rectal and sigmoid cancers account for up to $60 \%$ of all CRC cases [1]. Rectal carcinoma (RC) is the $8^{\text {th }}$ most common cancer in Kazakhstan ( $4.5 \%$ or 8.2 cases $/ 100,000$ population) and the $8^{\text {th }}$ leading cause of cancer-related mortality $(4.7 \%)$. Wherein, despite slight growth in CRC incidence by $0.5 \%$ during the 2015-2018 period, mortality rates decreased by $9.6 \%[2]$.

According to the National Comprehensive Cancer Network, the incidence of CRC per 100,000 population decreased from 60.5 in 1976 to 46.4 in 2005 and kept decreasing at a rate of $2.9 \%$ annually from 2005 to 2014 [3]. These improvements in incidence and mortality from CRC are believed to be a result of early diagnoses through population screening and implementing novel treatment modalities. At the same time, the incidence of CRC in pa-

Correspondence address:

Jandos Amankulov, Department of Radiology and Nuclear Medicine, Kazakh Institute of Oncology and Radiology, Abay Avenue 91, 050022 Almaty, Kazakhstan, e-mail:zhandos.amankulov@gmail.com

Authors' contribution:

A Study design · B Data collection · C Statistical analysis · D Data interpretation · E Manuscript preparation · F Literature search · G Funds collection 
tients younger than 50 years has been increasing. Similarly, incidence rates for CRC among the young population are expected to increase by $90.0-124.2 \%$ by $2030[1,3]$.

The role of imaging in CRC management has evolved greatly. Assessing the depth of tumour invasion, lymph node involvement and distant metastases determines the treatment option and overall prognosis. As of now, there is no consensus on preferred imaging modality for the final staging of RC is often a result of a complex approach with several imaging tools [4-6].

High-resolution magnetic resonance imaging (MRI) allows an excellent soft tissue contrast resolution, functional imaging ability, and multiplanar acquisition, assuring thorough RC evaluation [5-7]. Due to its ability to accurately evaluate the mesorectal fascia, anal sphincter, as well as mesorectal and pelvic lymph nodes, MRI has become a tool of choice for RC staging [8-10].

The purposes of this study are (i) depicting 3-Tesla (3T) MRI features of RC in correlation with histopathology results and (ii) assessment of its diagnostic accuracy for extramural tumour spread and involvement of lymph nodes.

\section{Material and methods}

\section{Patients}

This retrospective study was conducted at a leading incountry cancer care institution and included all $86 \mathrm{pa}-$ tients (46 men and 40 women, mean age $61.7 \pm 12.9$ years) with a newly diagnosed RC between January 2015 and June 2018. The study was approved by the institutional review board. The inclusion criterion was a new diagnosis of pathology-proven RC with no gender or age predilection. Patients who did not undergo the surgery and/or did not complete path evaluation, or did not obtain optimal imaging were excluded from the study.

\section{Magnetic resonance scan protocol}

Pre-operative MR imaging was performed on a $3 \mathrm{~T} \mathrm{MR}$ system (GE Discovery MR750w, USA), utilizing a pelvic phased-array surface coil (Gem body coil $8 \mathrm{ch}$.). The sequences and related parameters are listed in Table 1.

High-resolution, 2-dimensional, T2-weighted, fast spinecho (FSE) sequences in sagittal, axial, and coronal planes were used to create an imaging basis for the MR staging. The scanning started with the sagittal series, to plan the axial images, perpendicular to the rectal wall at the level of the tumour (to avoid volume averaging). Coronal images were oriented parallel to the anal canal for low rectal tumours to estimate the sphincter involvement, but parallel to the rectal wall in all other cases. Diffusion-weighted images were used to improve the imaging accuracy of tumour and lymph node involvement. T1-weighted images were used to assess concomitant changes and pelvic bones. MR examinations were performed without gadolinium administration.

\section{Image analysis}

The tumour was considered either as polypoid, an endophytic ulcerous, an exophytic circular, or as diffuse infiltrative type, based on the gross morphology. We have evaluated the tumour extension in centimetres $(\mathrm{cm})$ and the tumour level - the distance from anorectal transition to the lower border of the tumour - also in centimetres. Based on the latter, the level was considered as a lower, mid, or upper rectal tumour when the distance between lowest border of tumour and the anorectal junction is at 0-5 cm, 5-10 cm, or 10-15 cm, respectively.

The involvement of the circular resection margin is defined by measuring the distance from the border of the tumour or metastatic lymph nodes to the mesorectal fascia (MRF). The MRF was regarded as intact if the distance from the tumour margin was more than $2 \mathrm{~mm}$; a possible invasion was considered when the distance was 1-2 mm, and MSF was evaluated as positive at a distance less than $1 \mathrm{~mm}$ [11-14].

According to the TNM $7^{\text {th }}$ edition, lymph nodes (LN) are thought to be involved if greater than $5 \mathrm{~mm}$ in short axis, with other signs of malignancy including the heterogeneity, abnormal shape, and ill-defined or radiant contours [15-17]. The disease was considered N0 if no suspicious LN was found, N1 with 1-3 positive $\mathrm{LN}$, or $\mathrm{N} 2$ when 4 or more $\mathrm{LN}$ were involved.

Table 1. Magnetic resonance imaging sequences applied (3T) for preoperative rectal carcinoma staging

\begin{tabular}{|l|c|c|c|c|c|}
\hline \multirow{2}{*}{ Sequences } & \multicolumn{3}{|c|}{ T2 FSE-weighted imaging } & DWI & T1 FSE axial \\
\cline { 2 - 6 } & Sagittal & Axial & Coronal & & 650 \\
\hline TR (ms) & 7300 & 5500 & 8700 & 6000 & 9.4 \\
\hline TE (ms) & 90 & 120 & 100 & 90 & 50 \\
\hline Bandwidth (kHz) & 83.3 & 41.7 & 41,7 & 250 & 260 \\
\hline FOV (mm) & 260 & 260 & 260 & 260 & 4 \\
\hline Slice thickness (mm) & 4 & 4 & 4 & 4 & 0 \\
\hline Spacing (mm) & 0 & 0 & 0 & 0 & $320 \times 320$ \\
\hline Matrix & $320 \times 320$ & $320 \times 320$ & $320 \times 320$ & $128 \times 128$ & $2: 12$ \\
\hline Acquisition time (min) & $2: 45$ & $2: 35$ & $2: 15$ & $4: 36$ & \\
\hline
\end{tabular}

FSE - fast spin echo, DWI - diffusion-weighted imaging (b values were used: $0,1000,2000$ ), FOV - field of view 
The following groups of LN were assessed: within the mesorectum, extra-mesorectal locoregional lymph nodes (perirectal, sigmoid mesenteric, inferior mesenteric, lateral sacral, presacral, internal iliac, sacral promontory, internal iliac, superior, middle, or inferior rectal (hemorrhoidal). The involvement of other groups of pelvic LN and inguinal $\mathrm{LN}$ are regarded as distant metastases.

Extramural vascular invasion (EMVI) was assessed as the part of standard MR protocol [18-21].

\section{Tumour morphology and staging}

MRI reporting criteria for $\mathrm{T}$ and $\mathrm{N}$ staging of $\mathrm{RC}$ were listed as standard and described elsewhere [12-14]. Briefly, the tumour confined only to submucosa is considered as T1, with involvement of the muscular layer but sparing perirectal fat as T2, with mesorectal fat invasion as T3, and the extension to other pelvic organs or peritoneum as T4. Diffusion restriction $(b=1000)$ expressed by the apparent diffusion coefficient (ADC) was evaluated separately for adenocarcinoma, mucinous carcinoma, squamous cell carcinoma, and signet cell RC.

\section{Histopathological evaluation}

All patients underwent surgery. In all 86 patients, histological examination and pathological staging were performed on the surgical specimens. Seventy-one out of a total of 86 patients underwent preoperative neoadjuvant therapy consisting of radiotherapy and lor chemotherapy. For this group of cases MRI was performed before and after neoadjuvant treatment, but for pathology-radiology correlation post-treatment MRI data was used. Pathological staging was carried out according to TNM criteria [3]. A pathologist with 20 years of experience examined the specimens, blinded to the preoperative MR staging.

\section{Statistical analysis}

Correlative analysis of MRI and histopathological data was performed. The sensitivity, specificity, positive and

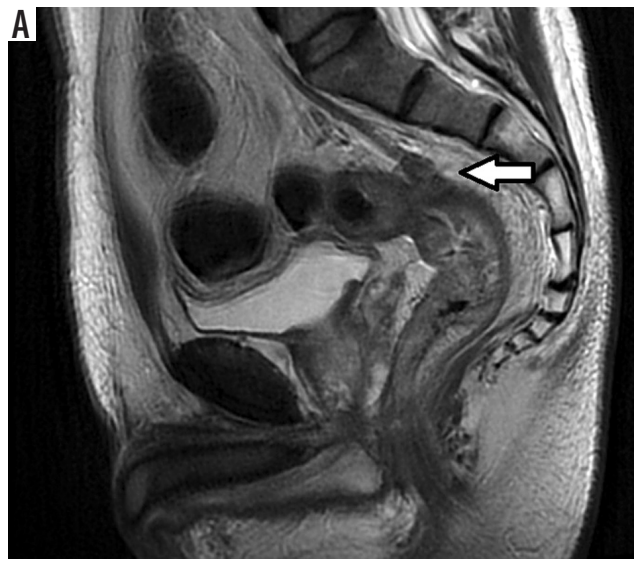

negative predictive values, and accuracy of MRI for T and $\mathrm{N}$ stages of RC were calculated with $95 \%$ confidence intervals (95\% CIs). The MRI was assessed by 2 radiologists with 7 and 15 years of experience in oncology imaging, respectively. Interobserver agreement was assessed using Cohen's $\kappa$ coefficient.

\section{Results}

\section{Tumour spread patterns}

Exophytic character of tumour growth was found in $63.95 \%(n=55)$ of cases, diffuse-infiltrative semi-circular growth in $18.6 \%(n=16)$, polypoid growth with/without a fibromuscular pedicle in $11.6 \%(n=10)$, and endophyticulcerous growth in $5.8 \%(n=5)$ of all samples.

The low rectum was the most prevalent site of cancer among all RC cases $(n=46,53.5 \%)$. The tumour was restricted within the lower rectum in $19(22.1 \%)$ cases, or in various combinations with the involvement of the anal canal and/or spread to the middle rectum $(n=27,31.4 \%)$. The middle rectum was affected in $34(39.5 \%)$ patients, while only in $10(11.6 \%)$ cases the tumour was restricted by the mid rectum. In the rest of the cases, the tumour invaded the adjacent parts of upper or lower rectum. The upper rectum on the whole was affected in 22 (25.6\%) cases, with the tumour affecting only the upper part of the rectum in $12(13.9 \%)$ patients. An advanced process of RC affecting all parts of the rectum was observed in 7 cases $(8.1 \%)$. The mean length of the lesions was $5.7 \pm 2.4 \mathrm{~cm}$ (range $2.2 \mathrm{~cm}$ to $15.6 \mathrm{~cm}$ ).

EMVI was identified in $14(16.2 \%)$ of all RC cases (Figure 1). The estimated diagnostic accuracy of MRI for EMVI as compared with tumour endovascular emboli evidence on pathology was $95 \%$.

\section{Correlation of rectal carcinoma imaging characteristics and histological profile}

The distribution of included RC cases by histological groups is presented in Table 2 . The majority of RC cases

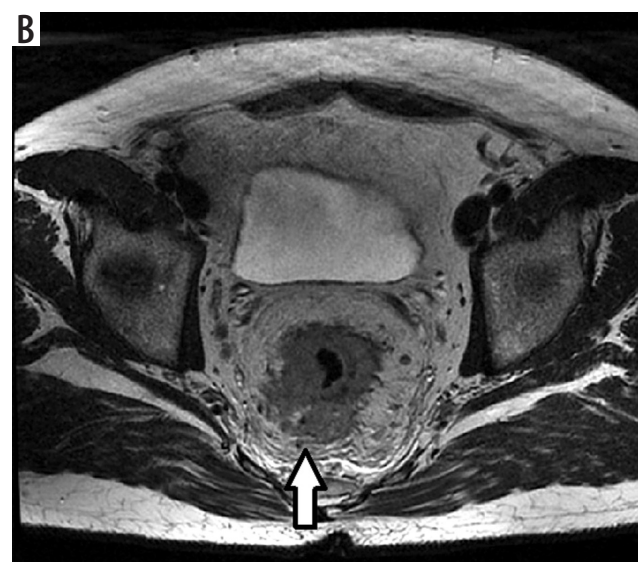

Figure 1. Extramural vascular invasion in G2 (A) and G3 (B) rectal carcinomas (arrows) 
Table 2. Comparison of histological data and magnetic resonance imaging features of rectal carcinoma

\begin{tabular}{|c|c|c|c|c|c|c|}
\hline \multirow[t]{3}{*}{ Parameter } & \multicolumn{6}{|c|}{ Histological form } \\
\hline & \multicolumn{3}{|c|}{ Adenocarcinoma } & \multirow{2}{*}{$\begin{array}{l}\text { Mucinous } \\
\text { adenocarcinoma }\end{array}$} & \multirow{2}{*}{$\begin{array}{l}\text { Squamous cell } \\
\text { carcinoma }\end{array}$} & \multirow{2}{*}{$\begin{array}{l}\text { Signet ring cell } \\
\text { carcinoma }\end{array}$} \\
\hline & G1 & G2 & G3 & & & \\
\hline$N=86(100 \%)$ & $3(3.5 \%)$ & $51(59.3 \%)$ & $7(8.1 \%)$ & $13(15.1 \%)$ & $8(9.3 \%)$ & $4(4.65 \%)$ \\
\hline$A D C b=1000\left(* 10^{-3} \mathrm{~mm}^{2} \backslash \mathrm{s}\right)$ & $0.846 \pm 0.17$ & $1.17 \pm 0.08$ & $0.91 \pm 0.11$ & $0.79 \pm 0.21$ & - & - \\
\hline Tumour size (cm) & $3.9 \pm 1.9$ & $5.7 \pm 2.5$ & $6.7 \pm 1.4$ & $7.2 \pm 2.7$ & $4.26 \pm 1.7$ & $8.1 \pm 1.6$ \\
\hline
\end{tabular}

Table 3. T-staging: comparison of magnetic resonance imaging data and histopathological examination

\begin{tabular}{|c|c|c|c|c|c|c|c|}
\hline \multirow[t]{2}{*}{ Path } & \multicolumn{6}{|c|}{ MRI } & \multirow{2}{*}{$\begin{array}{l}\text { Total } \\
\text { path }\end{array}$} \\
\hline & Tis & $\mathrm{T} 1$ & T2 & T3 & T4 & FN & \\
\hline Tis & - & - & 1 & - & - & 1 & 1 \\
\hline T1 & - & 3 & - & - & - & - & 3 \\
\hline T2 & - & 2 & 13 & 1 & - & 3 & 16 \\
\hline T3 & - & - & 3 & 34 & 5 & 8 & 42 \\
\hline T4 & - & - & - & 4 & 20 & 4 & 24 \\
\hline FP & - & 2 & 4 & 5 & 5 & 16 & \\
\hline TN & & 81 & 69 & 47 & 61 & - & \\
\hline Total & - & 5 & 17 & 39 & 25 & - & 86 \\
\hline
\end{tabular}

Path - pathomorphological data: $\mathrm{TN}$ - true negative, $\mathrm{FN}$ - false negative, $\mathrm{FP}$ - false positive

Table 4. N-staging: comparison of magnetic resonance imaging data and histopathological examination

\begin{tabular}{|l|c|c|c|c|}
\hline Path & \multicolumn{3}{|c|}{$\begin{array}{c}\text { MRI } \\
\text { N1-2-3 }\end{array}$} & $\begin{array}{c}\text { Total } \\
\text { path }\end{array}$ \\
\hline N0 & 10 & 6 & 6 & 16 \\
\hline N1-2-3 & 4 & 66 & 4 & 70 \\
\hline FP & 4 & 6 & 10 & - \\
\hline TN & 64 & 12 & - & - \\
\hline Total & 14 & 72 & - & 86 \\
\hline
\end{tabular}

Path - pathomorphological data, $\mathrm{TN}$ - true negative, $\mathrm{FN}$ - false negative, $\mathrm{FP}$ - false-positive

was a moderately differentiated (G2) adenocarcinoma ( $n=51,59.3 \%)$, and the rarest type was signet ring cell carcinoma $(n=4,4.65 \%)$. However, the signet ring cell carcinoma had greater tumour sizes $(8.1 \pm 1.6 \mathrm{~cm})$ in comparison with other RC histological types.
Mucinous adenocarcinoma was histologically verified in $13(15.1 \%)$ cases. Eight out of 13 patients with histologically proven mucinous adenocarcinoma cases were staged as T3 (61.5\%), 4 of them as T4 (30.8\%), and only 1 as T2 $(7.7 \%)$ at the moment of diagnosis. A significant difference in $\mathrm{ADC}$ was obtained for mucinous adenocarcinoma $\left(1.17 \pm 0.08 \times 10^{-3} \mathrm{~mm}^{2} / \mathrm{s}\right)$ when the ADC was less than $0.9 \times 10^{-3} \mathrm{~mm}^{2} / \mathrm{s}$ for all other histological groups $(p \leq 0.05)$.

\section{Magnetic resonance staging in comparison with histopathological data}

The results of pre- and postoperative $\mathrm{T}$ and $\mathrm{N}$ staging of are presented in Tables 3 and 4 .

The discrepancy between the MR and pathological T staging was noted in 16 cases out of 86. Pathomorphological examination found 1 case of Tis stage (villus adenoma with intramucosal foci of adenocarcinoma), while on MRI the lesion was described as a $\mathrm{T} 2$ rectal polypoid lesion. In 5 cases, MRI overestimated the involvement of adjacent pelvic organs with fibrosis after neoadjuvant therapy, but pathomorphology excluded the invasion of adjacent organs (T4 stage lowered to T3). In 2 cases we evaluated lesions as T1 stage on MRI data, while pathomorphology revealed the focus of invasion in the muscle layer, so T2 stage has been verified. In 3 cases the T2 stage of MRI was verified as pT3a during pathological examination. In $4 \mathrm{RC}$ cases the cT3 stage by MRI was changed as pT4 after pathomorphological study (in patients after neoadjuvant chemoradiation therapy).

The discrepancy between radiological study and histopathology for $\mathrm{N}$ staging was found in 10 cases out of 86. The agreement was defined as substantial (0.78) for $\mathrm{T}$ staging, moderate $(0.57)$ for $\mathrm{N}$ staging, and substantial for extramural vascular invasion assessment (0.63).

Table 5. Diagnostic value 3T magnetic resonance imaging in T staging of rectal carcinoma

\begin{tabular}{|l|c|c|c|c|c|}
\hline Stage & Sensitivity & Specificity & Positive predictive value & Negative predictive value & Accuracy \\
\hline T1 & $100 \%$ & $97.5 \%$ & $100 \%$ & $71.9 \%$ & $97.6 \%$ \\
\hline T2 & $81.3 \%$ & $94.5 \%$ & $95.3 \%$ & $78.5 \%$ & $92.1 \%$ \\
\hline T3 & $87.1 \%$ & $92.1 \%$ & $85.1 \%$ & $87.5 \%$ & $89.0 \%$ \\
\hline T4 & $83.3 \%$ & $92.4 \%$ & $93.1 \%$ & $81.9 \%$ & $90.0 \%$ \\
\hline Average & $87.9 \%$ & $94.1 \%$ & $93.4 \%$ & $80.0 \%$ & $92.2 \%$ \\
\hline
\end{tabular}


Table 6. Diagnostic value 3T magnetic resonance imaging in N staging of rectal carcinoma

\begin{tabular}{|l|c|c|c|c|c|}
\hline Stage & Sensitivity & Specificity & Positive predictive value & Negative predictive value & Accuracy \\
\hline N0 & $72.5 \%$ & $94.1 \%$ & $70.8 \%$ & $91.7 \%$ & $88.2 \%$ \\
\hline N 1,2,3 & $94.3 \%$ & $66.7 \%$ & $92.5 \%$ & $72.7 \%$ & $89.2 \%$ \\
\hline Average & $83.4 \%$ & $80.4 \%$ & $81.7 \%$ & $82.2 \%$ & $88.7 \%$ \\
\hline
\end{tabular}

The diagnostic accuracy of 3T MRI for different $\mathrm{T}$ and $\mathrm{N}$ stages of RC obtained in our work is presented in Tables 5 and 6 . The sensitivity and specificity of 3T MRI for the $\mathrm{T} 1$ stage was $100 \%$ and $97.5 \%$, respectively, for $\mathrm{T} 2$ it was $81.3 \%$ and $94.5 \%$, respectively, and for T3 stage it was $87.1 \%$ and $92.1 \%$, respectively.

T1 stage of RC without involvement of the muscular layer of the rectal wall was recorded in 5 patients $(5.8 \%)$ according to MR data, while T2 stage with involvement of the muscular layer was recorded in 17 patients (19.8\%). Most of the primary RC cases were staged as T3 - in 39 (45.3\%) patients, including 27 (31.4\%) cases without involvement of the mesorectal fascia (MRF-), and with a possible or obvious invasion (MRF+) in $12(14 \%)$ cases. Locally advanced tumour involving either pelvic peritoneum (T4a) or pelvic organs (T4c) was noted in $25(29.1 \%)$ cases. In the evaluation of metastatic lymph nodes, we obtained a total sensitivity of $83.4 \%$ and a specificity of $80.4 \%$.

\section{Discussion}

Our data revealed several patterns of tumour growth. More than a half of the RC cases had exophytic character of growth. In previously published data, exophytic growth pattern varied between 13.5 and 50\% for different CRC stages [35]. The lower third of the rectum was the part most commonly affected by RC in our study (53.5\%). According to the published data, the frequency of primary involvement of particular parts of the rectum varies, but it most often seen in the upper and middle third of the rectum $[13,14]$. Similarly, Brown et al. in 2006 reported the upper third vs. middle third rectal lesion in $34 \%$ and $36 \%$ of patients, respectively, while the lower lesion was seen only in $20 \%$ [6]. However, more often we observed an advanced process involving 2 or more parts of the rectum, with the predominant location being within the lower rectum.

EMVI is a known risk factor for early recurrence, tumour aggressiveness, and compromised sensitivity to neoadjuvant chemoradiotherapy $[18,19]$. It is also associated with poor prognosis of the course of disease and low overall survival [20-22]. The sensitivity and specificity of MRI in detection of EMVI were $62 \%$ and $88 \%$, respectively, in the study of Smith et al. in a 94-patient cohort [18]. Thus, MR-EMVI is used as a potential biomarker that facilitates the choice of method of treatment $[18,19]$. Sohn et al. proposed the MRI-detected EMVI as an independent risk factor for distant metastases; therewith the involvement of the large vessels (more than $3 \mathrm{~mm}$ in diameter) is associated with a higher risk in comparison with small vessels measuring less than $3 \mathrm{~mm}$ [19]. Similarly, Barbaro et al. showed the relationship of EMVI and risk of synchronous metastases in patients with a non-mucinous adenocarcinoma [21].

Mucinous adenocarcinoma has higher stage at the time of initial diagnosis, worse prognosis, and lower sensitivity to chemotherapy $[22,23]$. The tumour is considered mucinous if it contains at least $50 \%$ of the mucinous cells, wherein, mucinous components may be located in the submucosal or muscular layer and not be detected during colonoscopy $[7,8]$. Thus, the discovery of a mucus-forming tumour on MRI has clinical significance. In addition, some authors suggest a certain sampling error and underdiagnosis of mucinous component from histological biopsy given the heterogeneity of mixed forms of the lesions, which emphasizes the role of MRI in the differentiation of mucinous and non-mucinous forms of RC [7]. Attempts to differentiate mucinous from non-mucinous entities were proposed on the basis of increased T2 signal of rectal tumour as well
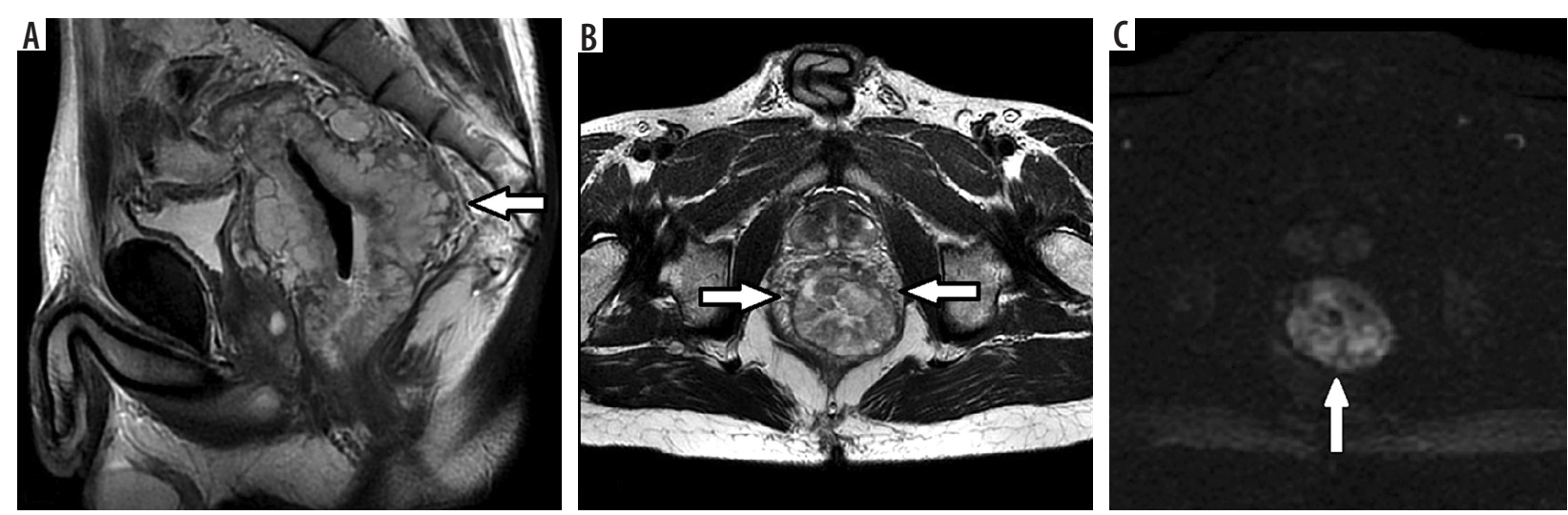

Figure 2. Rectal well-differentiated mucinous adenocarcinoma G1. High signal intensity of circular rectal tumour (arrows) with invasion of all layers of the rectal wall in T2 FSE sagittal (A) and axial (B) images. DWI with $b=1000$ (C) shows restricted diffusion within the tumour tissue (arrow), $A D C=1.7 \mathrm{~mm}^{2} \mid \mathrm{s} \times 10^{-3}$ 
as on the ADC value on DWI (Figure 2). It was shown that the diffusion coefficient is the most reliable factor for differentiation, whereas the sensitivity and specificity of T2 images are much lower [23].

However, according to the published data, there is still no consensus on the role of DWI. For example, inflammation within the submucosal layer of the rectal wall can lead to restricted diffusion and false positive results of DWI MRI [23-25]. There are studies supporting the use of diffusion-weighted mode to improve the diagnostic capabilities in assessing the local prevalence of rectal cancer and its recurrence. Thus, according to Balyasnikova et al., the use of DWI allows MRI specificity to be slightly increased, especially in patients receiving neoadjuvant therapy [8]. Barbaro et al. also reported significant differences in ADC values for mucinous and non-mucinous forms of rectal adenocarcinoma, and according to the positron emission tomography data from the same study, no significant differences of FDG uptake were obtained for these histological forms [22]. Çolakoğlu et al. reported statistically significant differences in the ADC for mucinous and non-mucosal adenocarcinoma [23]. There are also data showing that the direct estimation of DWI with calculation of ADC allows, with more than $90 \%$ specificity, prediction of the tumour response to radiation and chemotherapy [22,23]. However, according to Lambregts et al., DWI, including DWI-fused images, generally does not improve sensitivity and specificity of MR in mucinous RC staging [26]. A number of publications define ADC as a potential predictive biomarker that can assess the aggressiveness of a tumour [26-28].

The intensity of MR signals of the lesions at T2 FSE varied from homogeneous iso-intensive to hyperintense inhomogeneous. Diffuse or heterogeneous amplification of the MR signal on T2 images was considered for mucinous tumours $[22,23]$.

In similar research, the T3 stage was the dominant group in the studied cohort of patients (45-80\%) [29,30]. Thus, in the large multicentre MERCURY study [6], T3-stage was $45 \%, \mathrm{~T} 2-18 \%, \mathrm{~T} 1-8 \%$, and $\mathrm{T} 4-6 \%$. In the studies of Sani et al. and Chatterjee et al. $[13,14]$ the portion of T3 patients was $67 \%$ and $80 \%$, respectively. In the data of similar research, the distribution of patients within the groups according to such important criteria as involvement of mesorectal fascia (T3) or differences in the involvement of only the pelvic peritoneum or pelvic organs (T4 stage) is not given. At the same time, T4 stage was usually associated with involvement of pelvic organs; isolated lesion of pelvic peritoneum was much less common.

Pathomorphological examination found 1 case of Tis stage in our study. This case can be considered as casuistry, rarely observed in everyday practice; the recognition of the Tis stage lies outside the diagnostic capabilities of MRI. In this turn, when visualizing polypoid lesion, it is difficult to judge about the benign or malignant nature of the tumour based only on MR data.
In 5 cases, the involvement of adjacent pelvic organs after neoadjuvant therapy was overestimated and T4 stage was considered. This problem is often mentioned in similar previous studies $[14,29,30]$. Post-radiation fibrosis, as a rule, is usually present in patients with RC cases, which makes it difficult to determine the exact boundaries of the primary tumour and pre-operative re-staging. A possible solution to this problem is the use of DWI with a sufficiently high b-factor [24,33], and application of additional postcontrast series $[11,12]$, as well as the use of thin-cutting (1-2 mm) 3-dimensional T2 series [29,33].

Difficulties in recognizing the T1-T2 stage are often mentioned in the literature, especially in early studies based on 1.5T MRI [8-10]. Although many researchers did not notice significant differences in the sensitivity and specificity of $1.5 \mathrm{~T}$ and $3 \mathrm{~T}$ MRI in RC staging, 3T MRI provides a higher sensitivity in differentiation of the submucosal layer and the muscular wall $[8,29,30]$.

Mistakes in differentiation of T2 and T3a stages of RC (limited spread beyond the limits of muscularis propria plate to mesorectal fat) due to desmoplastic reaction and postradiation fibrosis are also often mentioned as one of the main reasons for decreased sensitivity and specificity of MRI [10-14,29,33]. In this case, the sensitivity for the T3 stage in these sources varied from 71 to $83 \%$, and the specificity from 20 to $76 \%$. Despite generally recognized limitations of MRI in separation of T2 and T3a, a clinical significance of this fact is small because the same therapeutic approach is used for these patients in many countries.

The detection of metastatic LN on MRI remains a difficult issue because of the multifactorial determinants of the involved nodes (size, margins, internal structure, and restriction of diffusion) $[10,16]$. On top of that, it is generally accepted that metastatic LNs in RC are usually small $(0.3-0.5 \mathrm{~cm})$ and it is hard to detect MR criteria of metastatic lesions [15-17]. Help in the differentiation of metastatic LNs is provided by the DWI technique, which is recommended by many authors $[24,27,31,32]$. However, the data on sensitivity and specificity of MRI for N-staging remain heterogeneous. Thus, in the study of Chatterjee et al. the sensitivity of MRI for N-criterion was $100 \%$ and the specificity was $78 \%$.

Wherein, a relatively low sensitivity of MRI for $\mathrm{N}_{0}$ stage was noticed $-72 \%$, with a sufficiently high specificity of $94 \%$, which indicates a potential overdiagnosis of metastatic LNs on MRI. Conversely, in the presence of metastatic nodes (N1-2), the pattern of the reliability of results is reversed - high sensitivity of $94 \%$ and low specificity of $66 \%$. Winter et al. reported about $95 \%$ and $91 \%$ accuracy of MR in $\mathrm{T}$ and $\mathrm{N}$ staging, respectively [30]. Kim et al. showed similar results in a group of 42 patients, where the diagnostic accuracy for $\mathrm{N}$ stage was $84-90 \%$ [33].

One of the important limitations of our study is the large variation of numbers of different $\mathrm{T}$ stages - a small number of Tis and T1 lesions but a large share of included T3 samples. Another limitation is that we did not divide the in- 
cluded RC cases to primary MR staging and MR staging after neoadjuvant treatment groups, and we did not analyse the diagnostic accuracy of 3T MRI for these groups separately.

\section{Conclusions}

Using a sufficiently large pool of cases, we assessed the diagnostic value of 3T MRI for both $\mathrm{T}$ and $\mathrm{N}$ staging, and we achieved fair accuracy of the results. According to our study, the diagnostic accuracy of $3 \mathrm{~T}$ MRI is $92.2 \%$ for the $\mathrm{T}$ staging and $88.7 \%$ for the $\mathrm{N}$ staging. The role of DWI in the pre-operative estimation of primary rectal tumour was proven to be significant, which allows the determination of a group of patients with high risk, and with more aggressive histology subtypes. Overall, 3T MRI using surface coils allows an accurate non-invasive diagnostic determination of rectal carcinoma.

\section{Conflict of interest}

The authors report no conflict of interest.

\section{References}

1. Arnold M, Sierra MS, Laversanne M, et al. Global patterns and trends in colorectal cancer incidence and mortality. Gut 2017; 66: 683-691.

2. Oncology service statistics review (statistic data of Kazakh State Institution of Oncology and Radiology)/under rev. of Prof. Kaidarova DR, Almaty; 2018.

3. Cheng L, Eng C, Nieman LZ, et al. Trends in colorectal cancer incidence by anatomic site and disease stage in the United States from 1976 to 2005. Am J Clin Oncol 2011; 34: 573-580.

4. Benson AlB, Vernook AP. Rectal cancer, Version 2.2018. Clinical practice guidelines in oncology. J Natl Compr Canc Netw 2018; 16: 874-901.

5. Hricak H, Husband J, Panicek DM. Oncologic Imaging: Essentials of Reporting Common Cancers. Translated under rev. of Tuyrin IE. M: Practical Medicine; 2014, p. 42-49.

6. Heo SH, Kim JW, Shin SS, et al. Multimodal imaging evaluation in staging of rectal cancer. World J Gastroenterol 2014; 20: 4244-4255.

7. MERCURY Study Group. Diagnostic accuracy of preoperative magnetic resonance imaging in predicting curative resection of rectal cancer: prospective observational study. BMJ 2006; 333: 779.

8. MERCURY Study Group. Extramural depth of tumor invasion at thin-section MR in patients with rectal cancer: results of the MERCURY study. Radiology 2007; 243: 132-139.

9. Balyasnikova SS, Suraeva YE, Dolgushin BI, et al. The role of magnetic resonance imaging in the assessment of the rectal carcinoma local spread. Coloproctology 2014; 1: 4-13.

10. Berezovskaya TP, Nevolskih AA, Berdov BA, Shavladze ZN. Diag nostic capabilities of the magnetic resonance imaging in rectal carcinoma. Coloproctology 2014; 1: 14-21.

11. Taylor FG, Swift RI, Blomqvist L, Brown G. A systematic approach to the interpretation of preoperative staging MRI for rectal cancer. AJR Am J Roentgenol 2008; 191: 1827-1835.

12. Tapan Ü, Özbayrak M, Tatlı S. MRI in local staging of rectal cancer: an update. Diagn Interv Radiol 2014; 20: 390-398.

13. Jhaveri KS, Hosseini-Nik H. MRI of rectal cancer: an overview and update on recent advances. AJR Am J Roentgenol 2015; 205 : W42-W55.

14. Chatterjee P, Eapen A, Perakath B, Singh A. Radiologic and pathological correlation of staging of rectal cancer with 3 Tesla magnetic resonance imaging. Can Assoc Radiol J 2011; 62: 215-222.

15. Sani F, Foresti M, Parmiggiani A, et al. 3-T with phased array surface coil in the local staging of rectal cancer. Radiol Med 2011; 116: 375-388.
16. Wang C, Zhou Z, Wang Z, et al. Patterns of neoplastic foci and lymph node micrometastasis within the mesorectum. Langenbecks Arch Surg 2005; 390: 312-318.

17. Kim JH, Beets GL, Kim MJ, et al. High-resolution MR imaging for nodal staging in rectal cancer: are there any criteria in addition to the size? Eur J Radiol 2004; 52: 78-83.

18. Brown G, Richards CJ, Bourne MW, et al. Morphologic predictors of lymph node status in rectal cancer with use of high-spatial-resolution MR imaging with histopathologic comparison. Radiology 2003; 227: 371-377.

19. Smith NJ, Barbachano Y, Norman AR, et al. Prognostic significance of magnetic resonance imaging-detected extramural vascular invasion in rectal cancer. Br J Surg 2008; 95: 229-236.

20. Sun Y, Li J, Wang X, Tong T, Gu Y. Predictive value of MRI-detected extramural invasion in stage $\mathrm{N} 3$ rectal cancer patients before neoajuvant chemoradiation. Diagn Interv Radiol 2018; 24: 128-134.

21. Tripathi P, Rao SX, Zeng MS. Clinical value of MRI-detected extramural venous invasion in rectal cancer. J Dig Dis 2017; 18: 2-12.

22. Sohn B, Lim JS, Kim H, et al. MRI-detected extramural vascular invasion is an independent prognostic factor for synchronous metastasis in patients with rectal cancer. Eur Radiol 2015; 25: 1347-1355.

23. Barbaro B, Leccisotti L, Vecchio FM, et al. The potential predictive value of MRI and PET-CT in mucinous and nonmucinous rectal cancer to identify patients at high risk of metastatic disease. Br J Radiol 2017; 90: 20150836.

24. Çolakoğlu Er H, Erden A. Mean ADC values discriminate rectal mucinous carcinoma from rectal nonmucinous adenocarcinoma. Turk J Med Sci 2017; 47: 1520-1525.

25. Bauerle T, Seyler L, Munter M, et al. Diffusion weighted imaging in rectal carcinoma patients without and after chemoradiotherapy: a comparative study with histology. Eur J Radiol 2013; 82: 444-452.

26. Monguzzi L, Ippolito D, Bernasconi DP, et al. Locally advanced rectal cancer: value of ADC mapping in predict of tumor response to radiochemotherapy. Eur J Radiol 2013; 82: 234-240.

27. Lambregts D, Cappendijk V, Maas M, et al. Value of MRI and diffusion-weighted MRI for the diagnosis of locally recurrent rectal cancer. Eur Radiol 2011; 21: 1250-1258.

28. Curvo-Semedo L, Lambregts DM, Maas M, et al. Diffusion-weighted MRI in rectal cancer: apparent diffusion coefficient as a potential noninvasive marker of tumor aggressiveness. J Magn Reson Imaging 2012; 35: 1365-1371. 
29. Hunter CJ, Garant A, Vuong T, et al. Adverse features on rectal MRI identify a high-risk group that may benefit from more intensive preoperative staging and treatment. Ann Surg Oncol 2012; 19: 1199-1205.

30. Futterer JJ, Yakar D, Strijk SP, et al. Preoperative 3T MR imaging of rectal cancer: local staging accuracy using a two-dimensional and three-dimensional T2-weighted turbo spin echo sequence. Eur J Radiol 2008; 65: 66-71.

31. Winter L, Bruhn H, Langrehr J, et al. Magnetic resonance imaging in suspected rectal cancer: determining tumor localization, stage, and sphincter-saving resectability at 3-Tesla-sustained high resolution. Acta Radiol 2007; 48: 379-387.
32. Altman DG, Bland JM. Diagnostic tests 1: sensitivity and specificity. BMJ 1994; 308: 1552.

33. Karmazanovskii GG. Estimation of the diagnostic method value ("sensitivity", "specificity" and "general accuracy"). Ann Surg Hepatol 1997; 2: 139-142.

34. Kim SH, Lee JM, Lee MW, et al. Diagnostic accuracy of 3.0-Tesla rectal magnetic resonance imaging in preoperative local staging of primary rectal cancer. Invest Radiol 2008; 43:587-593.

35. Papagiorgis PC, Zizi AE, Tseleni S, et al. Clinicopathological differences of colorectal cancers according to tumor origin: Identification of possibly de novo lesions. Biomed Rep 2013; 1: 97-104. 